\title{
Realising the benefits of data driven digitalisation without ignoring the risks: health data governance for health and human rights
}

\author{
Stefan Germann, Ursula Jasper \\ Fondation Botnar, Basel, Switzerland \\ Correspondence to: Ursula Jasper. Policy Officer Fondation Botnar, Basel, Switzerland. Email: ujasper@fondationbotnar.org.
}

Received: 30 January 2020; Accepted: 30 June 2020; Published: 05 October 2020.

doi: 10.21037/mhealth-2019-di-11

View this article at: http://dx.doi.org/10.21037/mhealth-2019-di-11

Data driven digitalisation, including artificial intelligence (AI) and machine learning, is enabling a fundamental and deep transformation of healthcare systems, health services and medical practices, primarily in high income countries, and increasingly in low- and middle-income countries (LMICs). However, we suggest more attention must be paid to ensuring digital technologies do not reinforce or introduce new forms of inequalities in which its potential is fully realised only in certain regions, countries or patient groups.

The transformative power of digital technology is undeniable. A recent assessment of the impact of digital technologies on health services suggests that improved digital knowledge and diagnostic, preventative, treatment and rehabilitation possibilities have substantially altered healthcare systems and service provision, especially in terms of structure, culture, professions, treatments and outcomes (1). Internet connected devices (e.g., the Internet of Things) continue to surge and improve and together with 'big data' are becoming key inputs for innovation, research and development to address current and emerging health challenges (2).

In economic terms, it is estimated that by 2021 the market for AI health applications will reach up to USD 6.6 billion-11 times larger than in 2014 (3). In the United States, for example, the application of AI could contribute to USD 150 billion in saved healthcare costs annually by 2026 (3). Importantly, the potential impact of digital technology and AI is not limited to wealthy countries. Socalled "frontier technologies" also offer transformative opportunities for reaching universal health coverage (UHC) in LMICs with relatively low upfront costs (4-6). For example, the introduction of mobile phones to remote areas of Africa has allowed for the 'leapfrogging' of intensive landline infrastructure to realise the social and economic benefits of telephone networks (5,7). Moreover, these technologies are contributing to vast improvements in patient diagnostics, real-time remote monitoring and disease surveillance and increasing access to timely and appropriate care [1-3 (8)]. In fact, numerous international organisations, expert panels and advocacy groups assert that by leveraging the benefits of modern technology we can achieve significant progress towards UHC by 2030-a goal that the United Nations (UN) member states set themselves with the UN Sustainable Development Agenda (4).

But without intention, this new technology may just build on existing inequalities. Issues such as technical feasibility must be addressed, including the massive digital divide in internet connectivity that still prevails in many resource-poor settings. It is estimated that an additional USD 100 billion would be needed to achieve universal, affordable, and good quality broadband internet access in Africa by 2030 (9). The right policies and institutions must also be in place to take advantage of frontier technologies, such as building the necessary system and human capacities, including digital mindsets, to support the reimagining of health in a digital age (10).

As many of the papers in this series show, technological developments such as AI, innovative mobile phone applications, social media interventions, algorithm-based image recognition or interactive voice response software, can only advance with the use of big data, which is in turn influencing how data is stored, accessed, shared and used in health practice and research contexts. For example, access to and use of data has become essential for decision making in public health at the local, national, and global 
levels. This includes individual health data (e.g., electronic medical records, prescriptions, personal insurance data), health systems data (claims and payments data) and routine public health data (e.g., civil registration and vital statistics). These data sources create opportunities for previously unimaginable applications of data collection, processing and analysis. In addition to their primary use by public health agencies, public health data have become valuable for secondary use such as by academic researchers and for technological development (10). Interlinking, connecting and networking these different data sources could help to generate a more comprehensive understanding of health conditions and issues, and strengthen the capability to predict disease, intervene and provide preventative measures based on algorithmically-derived probabilities $(7,11)$.

Although the collection and processing of unprecedented amounts of health data is rich with potential and provides the fuel for much of the ongoing technological transformation, data governance models have been unable to keep pace (12). A lack of comprehensive political, legal and regulatory frameworks means current digital health research and development takes place in a largely autonomous "Wild West"-like landscape, that is characterized by a seemingly natural, almost constant extraction and appropriation of health data from individuals and even societies (13). Others claim this unregulated landscape resembles the colonial narrative of the "no man's land"-an uninhabited territory that is "available for exploitation without legal interference" (14). Governance mechanisms need to be established in response, at national and global levels, to balance the ethical concerns and rights of individuals and societies with the potential offered by big data in health. Here we give attention to two interlinked dimensions of health data governance: the need to improve the sharing of high-quality health data while at the same time ensuring that the benefits of big data are not achieved at the expense of data protection and privacy rights.

First, within the growing digital economy, data sharing is an essential practice, whether within an organisation, between partners and stakeholders or even, as in growing open data movements, with the public. Data sharing facilitates transparency and cooperation, replicability of research methods, cost-efficiency and preventing duplication, and the acceleration of discovery and innovation (10). Despite global commitments to the use and sharing of public health data, a range of challenges exist, including technical, motivational, economic, political, legal, and ethical barriers, such as security and privacy risks (10). Technical barriers in particular are well recognised as part of systemic challenges in health information system capacity in many LMICS. As a result, much healthcare data is generated in high-income settings which in turn creates skewed or inadequate datasets with the potential of bias (15).

Second, protecting privacy and data ownership is a complex challenge in health data governance. From a human rights perspective the most vulnerable in society are increasingly subject to demands and forms of intrusion without accountability, with citizens' information becoming much more accessible to private companies and governments, instead of the other way around (16). This presents a potential "new structure of power over individuals" (16). Individuals may not know how their data is being collected, processed or repurposed, or that the results could potentially discriminate against the individuals and communities concerned $(17,18)$. A lack of transparency in the creation and deployment of algorithms can result in inbuilt discrimination and bias, including related to gender, health and economic status. For example, an algorithm designed to support skin cancer diagnostics will be flawed if it is based on skewed, non-representational datasets that insufficiently mirror geographic, ethnic or gender diversity $(15,19)$. Many datasets also exist in silos and are not publicly available, despite being publicly funded. This is an impediment to the realization of new knowledge and prevents health data flowing back to society to strengthen the health system for everyone.

Ownership of patient data is a key area that must be tackled before equity can be secured. Current legal provisions in many countries do not sufficiently address the full range of ethical issues related to data protection, privacy and data misuse. There is also a lack of accountability systems to ensure clear responsibility for data ownership and data use, the consequences on individuals, families and communities (20) and mechanisms for remedy and redress as required. An important step would be for governments to support the creation of AI-based health data ecosystems that not only guarantee privacy and informed consent for data use, but ultimately achieve a more beneficial tradeoff between data privacy and societal utility. Here, new technological solutions, such as federated or split learning, can support higher levels of privacy and data security and thereby foster public trust in health data sharing $(21,22)$. For example, initiatives such as federated learning hospital networks are training AI models using local datasets for clinical areas such as cancer, dementia and stroke. These can then be shared with a centralized server to create a dataset across hospitals to form a reference point for clinical 
research. As the model is trained during multiple iterations at different sites it removes the need to pool data in a single location and ensures the protection of patients' privacy (23). Indeed, if we can achieve expanded, high-quality datasets, and public trust in sharing can be secured, we are likely to see an increase in well-functioning, less-biased, and more effective data-based applications and AI algorithms.

The opportunities to harness the transformative power of big data have never been greater but solutions to data health governance challenges are urgently needed. At Fondation Botnar, we are prioritizing the development and implementation of digital solutions, particularly AI, to improve the health and wellbeing of young people (between 10 and 24 years of age) in LMICs, by supporting innovative approaches in countries to involving young people themselves in the design of solutions. For example, together with partners Fondation Botnar has founded a coalition to support Young Experts: Tech 4 Health (YE:T4H), a platform for young people to shape and guide the agenda towards achieving Universal Health Coverage (UHC) by 2030 through data and digital technologies (24). As innovations move from pilot to scale, we must work to reduce the fragmentation of data, collaborate more to avoid the unnecessary duplication of technological capabilities and improve the sharing of valuable, anonymized data that might benefit the public good. These efforts as well as the other issues touched on above would benefit from regulatory measures for data collection, storage, sharing and ownership. It underscores the necessity to establish a global regulatory framework that aligns with and protects the needs of all stakeholders-individuals, governments, research institutes, the private sector and other constituency groups and organizations-as well as the rights and interests of the young people that Fondation Botnar is striving to support. Such a global mechanism could facilitate the evolution of a regulated health data ecosystem, guaranteeing information security and the right to privacy, while allowing for comprehensive data collection, analysis, and sharing.

We therefore welcome ongoing global efforts such as the UN Secretary's High-Level Panel on Digital Collaboration (6), the recently established Lancet and Financial Times Commission 'Governing Health Futures 2030: Growing up in a Digital World' (12) and the World Health Organization's draft Global Strategy on Digital Health (4), for catalysing global, cross-sectoral and inclusive dialogue and analysis on this pressing issue. We also call attention to the role of young people who, as the next generation of digital natives, must have an opportunity to participate in shaping a more fair and equitable digital future.

\section{Acknowledgments}

Funding: None.

\section{Footnote}

Provenance and Peer Review: This article was commissioned by the Guest Editors (Carinne Brody and Sarah Sullivan) for the series "Digital Interventions for Hard-to-reach Populations" published in mHealth. The article did not undergo external peer review.

Conflicts of Interest: Both authors have completed the ICMJE uniform disclosure form (available at http:// dx.doi.org/10.21037/mhealth-2019-di-11). The series "Digital Interventions for Hard-to-reach Populations" was commissioned by the editorial office without any funding or sponsorship. The authors have no other conflicts of interest to declare.

Ethical Statement: The authors are accountable for all aspects of the work in ensuring that questions related to the accuracy or integrity of any part of the work are appropriately investigated and resolved.

Open Access Statement: This is an Open Access article distributed in accordance with the Creative Commons Attribution-NonCommercial-NoDerivs 4.0 International License (CC BY-NC-ND 4.0), which permits the noncommercial replication and distribution of the article with the strict proviso that no changes or edits are made and the original work is properly cited (including links to both the formal publication through the relevant DOI and the license). See: https://creativecommons.org/licenses/by-nc-nd/4.0/.

\section{References}

1. Expert Panel on effective ways of Investing in Health. Assessing the impact of digital transformation of health services. Luxembourg: European Union, 2019.

2. Paunov C, Planes Satorra S. How are digital technologies changing innovation. OECD Science, Technology and Industry Policy Papers. No.74: OECD2019. Available online: https://www.oecd-ilibrary.org/science-andtechnology/how-are-digital-technologies-changinginnovation_67bbcafe-en 
3. Collier M, Fu R, L. Y. Artificial intelligence: healthcare's new nervous system: Accenture 2017. Available online: https://www.accenture.com/_acnmedia/pdf-49/accenturehealth-artificial-intelligence.pdf

4. World Health Organization. Draft global strategy on digital health 2020-2024. Geneva: World Health Organization 2019.

5. Zhenmin L. Frontier Technologies: A window of opportunity for leapfrogging!: United Nations 2019.

6. UN Secretary General's High-Level Panel on Digital Cooperation. The Age of Digital Interdependence 2019.

7. Germann S, Fombu E, Gitahi G, et al. Africa: Why Harnessing Digital Tech For Universal Health Coverage is Essential. All Africa 2019. Available online: https:// allafrica.com/stories/201912120196.html

8. Olu O, Muneene D, Bataringaya JE, et al. How Can Digital Health Technologies Contribute to Sustainable Attainment of Universal Health Coverage in Africa? A Perspective. Front Public Health 2019;7:341.

9. Broadband Commission for Sustainable Development. Connecting Africa Through Broadband. A strategy for doubling connectivity by 2021 and reaching universal access by 2030. 2019. Available online: https://www. broadbandcommission.org/Documents/working-groups/ DigitalMoonshotforAfrica_Report.pdf

10. van Panhuis WG, Paul P, Emerson C, et al. A systematic review of barriers to data sharing in public health. BMC Public Health 2014;14:1144.

11. Balicer RD, Afek A. Digital health nation: Israel's global big data innovation hub. Lancet 2017;389:2451-3.

12. Kickbusch I, Agrawal A, Jack A, et al. Governing health futures 2030: growing up in a digital world-a joint The Lancet and Financial Times Commission. Lancet 2019;394:1309.

13. Nebeker C, Torous J, Bartlett Ellis RJ. Building the case

doi: 10.21037/mhealth-2019-di-11

Cite this article as: Germann S, Jasper U. Realising the benefits of data driven digitalisation without ignoring the risks: health data governance for health and human rights. mHealth 2020;6:34. for actionable ethics in digital health research supported by artificial intelligence. BMC Med 2019;17:137.

14. Couldry N, Mejias U. Data colonialism: rethinking big data's relation to the contemporary subject. Television and New Media 2019;20. DOI: 10.1177/1527476418796632

15. Zou J, Schiebinger L. AI can be sexist and racist - it's time to make it fair. Nature 2018;559:324-6.

16. Kickbusch I. The dark side of digital health. BMJ 2020. Available online: https://blogs.bmj.com/bmj/2020/01/14/ ilona-kickbusch-the-dark-side-of-digital-health/

17. Hand DJ. Aspects of data ethics in a changing world: Where are we now?. Big Data 2018;6:176-90.

18. Information Commissioner's Office. Big data, artificial intelligence, machine learning and data protection. United Kingdom Information Commissioner's Office 2017.

19. Char DS, Shah NH, Magnus D. Implementing Machine Learning in Health Care - Addressing Ethical Challenges. N Engl J Med 2018;378:981-3.

20. Vayena E, Haeusermann T, Adjekum A, et al. Digital health: meeting the ethical and policy challenges. Swiss Med Wkly 2018;148:w14571.

21. Raskar R, Asthana A, Vidwans S, et al. AI for Health: Global Opportunity for Data Transparent Health Ecosystem 2019. doi: 10.13140/RG.2.2.30304.53765.

22. Choudhury O, Gkoulalas-Divanis A, Salonidis T, et al. Differential Privacy-enabled Federated Learning for Sensitive Health Data 2019:arXiv:1910.02578.

23. AI Powered Healthcare. UK Alliance to build 'federated learning' hospital network. 2019. Available online: https://www.healthcareitnews.com/ai-poweredhealthcare/uk-alliance-build-\%E2\% 80\%98federatedlearning\%E2\% 80\%99-hospital-network-0.

24. Plan International. Young Experts: Tech 4 Health. 2019. Available online: https://plancanada.ca/yet4h. 